Portland State University

PDXScholar

8-1-1971

\title{
On the Molecular Theory of Dielectric Polarization in Rigid-Dipole Fluids
}

John D. Ramshaw

Portland State University, jdramshaw@yahoo.com

Follow this and additional works at: https://pdxscholar.library.pdx.edu/phy_fac

Part of the Physics Commons

Let us know how access to this document benefits you.

\section{Citation Details}

J.D. Ramshaw, "On the Molecular Theory of Dielectric Polarization in Rigid-Dipole Fluids," J. Chem. Phys. $55,1763(1971)$

This Article is brought to you for free and open access. It has been accepted for inclusion in Physics Faculty Publications and Presentations by an authorized administrator of PDXScholar. Please contact us if we can make this document more accessible: pdxscholar@pdx.edu. 


\title{
On the Molecular Theory of Dielectric Polarization in Rigid-Dipole Fluids*
}

\author{
JoHN D. Ramshaw $\dagger$ \\ Institute for Fluid Dynanics and Applied Mathematics, University of Maryland, College Park, Maryland 20742
}

(Received 16 April 1971)

\begin{abstract}
A molecular theory is developed for the polarization $\mathbf{P}(\mathbf{r})$ produced by a weak position-dependent external electric field $\mathbf{E}_{0}(\mathbf{r})$ in a finite fluid system, of arbitrary shape, composed of rigid polar molecules. The theory differs from earlier work in that no assumption is made concerning the form of the electrostatic constitutive relation. [The usual assumption in this regard is that $\mathbf{P}(\mathbf{r})=(\epsilon-1) \mathbf{E}(\mathbf{r}) / 4 \pi$, where $\mathbf{E}(\mathbf{r})$ is the total Maxwell electric field. The "dielectric constant" $\epsilon$ is well defined only if the relation between $\mathbf{P}(\mathbf{r})$ and $\mathbf{E}(\mathbf{r})$ is in fact one of constant local proportionality.] The result of the present theory is a nonlocal relation between $\mathbf{P}(\mathbf{r})$ and the external field $\mathbf{E}_{0}(\mathbf{r})$. The contribution to $\mathbf{P}\left(\mathbf{r}_{1}\right)$ produced by $\mathbf{E}_{0}\left(\mathbf{r}_{2}\right)\left(\mathbf{r}_{1} \neq \mathbf{r}_{2}\right)$ is determined by the orientational correlation which exists in zero applied field between two representative dipoles located at $\mathbf{r}_{1}$ and $\mathbf{r}_{2}$. In principle this result may be used to investigate the conditions under which the dielectric constant is well defined, a question of considerable interest but one which has received little attention. The probable existence of long-range orientational correlations in polar fluids unfortunately precludes at present such an investigation for dense fluids, although for dilute gases the investigation can proceed by means of a density expansion. In this way it is demonstrated that the dielectric constant is well defined at least to second order in the density. This demonstration provides some insight into the connection between long-range dipolar effects on the macroscopic and molecular levels. It also yields automatically expressions for the first and second "dielectric virial coefficients"; these expressions agree with results obtained by previous workers under the assumption that the dielectric constant is well defined.
\end{abstract}

\section{INTRODUCTION}

When a dielectric is subjected to an applied electric field, a relative displacement of its constituent positive and negative charges occurs; this phenomenon is called dielectric polarization. The macroscopic behavior of a polarized dielectric may be interpreted by attributing an induced dipole moment $\mathbf{P}(\mathbf{r}) d^{3} \mathbf{r}$ to each of its volume elements $d^{3} \mathbf{r}$. The function $\mathbf{P}(\mathbf{r})$ of the position $\mathbf{r}$ is termed the polarization. If $\mathbf{P}(\mathbf{r})$ is known, the macroscopic Maxwell equations may be solved in terms of it for the macroscopic Maxwell electric field $\mathrm{E}(\mathbf{r})$, which is just the sum of the applied field and the field produced by $\mathbf{P}(\mathbf{r})$. The solution may be written in the form ${ }^{1,2}$

$$
\begin{aligned}
\mathbf{E}(\mathbf{r})=\mathbf{E}_{0}(\mathbf{r})- & (4 \pi / 3) \mathbf{P}(\mathbf{r}) \\
& +\lim _{\delta \rightarrow 0} \int_{\left|\mathbf{r}-\mathbf{r}^{\prime}\right|>\delta} d^{3} \mathbf{r}^{\prime} \mathbf{T}\left(\mathbf{r}-\mathbf{r}^{\prime}\right) \cdot \mathbf{P}\left(\mathbf{r}^{\prime}\right),
\end{aligned}
$$

where $E_{0}(\mathbf{r})$ is the externally-applied electric field acting to polarize the dielectric, $\mathbf{T}(\mathbf{r})$ is the familiar dipole tensor

$$
T(\mathbf{r}) \equiv \nabla \nabla|\mathbf{r}|^{-1}=-\left(|\mathbf{r}|^{-3} \mathbf{U}-3|\mathbf{r}|^{-5} \mathbf{r r}\right)
$$

and $\mathbf{U}$ is the unit dyadic. The inequality $\left|\mathbf{r}-\mathbf{r}^{\prime}\right|>\delta$ beneath the integral sign in Eq. (1) means that the integration variable $\mathbf{r}^{\prime}$ is excluded from the region within the sphere $\left|\mathbf{r}-\mathbf{r}^{\prime}\right|=\delta$. The limit $\delta \rightarrow 0$ in Eq. (1) is to be understood to mean that $\delta$ becomes much smaller than any macroscopic length but remains much larger than lengths of molecular size.

If $\mathbf{r}$ lies within the dielectric, the integral in Eq. (1) is improper; this is why it is necessary to exclude a cavity at the point $\mathbf{r}^{\prime}=\mathbf{r}$ from the range of the integration. As is well known, the value of this integral depends upon the shape of the cavity. The term $-4 \pi P(r) / 3$ in Eq. (1) is a consequence of our use of a spherical cavity. If a needle-shaped cavity with axis along $\mathbf{P}(\mathbf{r})$ were adopted, the term $-4 \pi \mathbf{P}(\mathbf{r}) / 3$ would be incorporated into the integral and would no longer appear explicitly. For our purposes, however, the spherical cavity is more convenient.

The externally-applied field $\mathbf{E}_{0}(\mathbf{r})$ will be left arbitrary throughout our discussion, except for the following restrictions: (a) The sources of $\mathbf{E}_{0}(\mathbf{r})$ are assumed to be external to the dielectric and to be held fixed. (b) The spatial variation of $E_{0}(\mathbf{r})$ with $\mathbf{r}$ is slow in a molecular sense; that is, $E_{0}(r)$ is essentially constant throughout any region of molecular size. (c) $\mathbf{E}_{0}(\mathbf{r})$ is sufficiently weak that $\mathbf{P}(\mathbf{r})$ is, to an excellent approximation, linearly related to it.

It is desirable to consider with some care exactly what takes place when a dielectric is polarized by an external field. Let us visualize a sample of some particular dielectric fluid, at given temperature and density, which occupies a region of volume $V$ and shape $S$ surrounded by vacuum. We now turn on an external field $\mathbf{E}_{0}(\mathbf{r})$ and wait for equilibrium to be reestablished. We find that a polarization $\mathbf{P}(\mathbf{r})$ has appeared at each point $\mathbf{r}$ within the sample. It is clear that $\mathbf{P}(\mathbf{r})$ is uniquely determined by the sample material, its temperature and density, the volume and shape of the sample, and the external field $\mathbf{E}_{0}(\mathbf{r})$; these will be referred to for short as the "given quantities" of the problem. Since $\mathbf{P}(\mathbf{r})$ is uniquely determined, the Maxwell electric field $\mathbf{E}(\mathbf{r})$ is also uniquely determined; it is given in terms of $\mathbf{P}(\mathbf{r})$ by $\mathbf{E q}$. (1). But although both $\mathbf{P}(\mathbf{r})$ and $\mathbf{E}(\mathbf{r})$ are uniquely determined by the given quantities of the problem, neither one is known a priori. We can obtain $\mathbf{E}(\mathbf{r})$ from $\mathbf{P}(\mathbf{r})$ by using $\mathrm{Eq}$. (1), but as yet we have no way of obtaining $\mathbf{P}(\mathbf{r})$. The determination of $\mathbf{P}(\mathbf{r})$ from the given quantities which 
determine it may be regarded as the central problem of dielectric theory.

Equation (1) already provides us with all the information which can be obtained from the Maxwell equations, for they serve only to tell one what the field is for a given distribution of sources. The determination of the sources themselves [in the present case $\mathbf{P}(\mathbf{r})$ ] is another matter entirely, and requires additional information. The problem may be looked at in the following way: Equation (1), which is equivalent to the Maxwell equations, is only one equation in the two unknowns $\mathbf{P}(\mathbf{r})$ and $\mathbf{E}(\mathbf{r})$, and must therefore be supplemented with another relation between them before either can be determined. It is clear that such a relation exists because if we set up experimentally the conditions we have described, Nature will intervene and impose a unique solution on the problem. The missing relation between $\mathbf{P}(\mathbf{r})$ and $\mathbf{E}(\mathbf{r})$ is called the electrostatic constitutive relation because it depends on the nature (or "constitution") of the particular dielectric substance in question. [However, it may conceivably depend also on the other given quantities of the problem, such as the shape of the sample or the functional form of $E_{0}(\mathbf{r})$.] From this point of view, the obstacle to our obtaining $\mathbf{P}(\mathbf{r})$ [and, from it, $\mathbf{E}(\mathbf{r})]$ is the fact that we do not know the form of the electrostatic constitutive relation, and the central problem of dielectric theory may be redefined as that of determining it.

It has long been customary to avoid this problem by assuming that the electrostatic constitutive relation consists of a simple local proportionality between $\mathbf{P}(\mathbf{r})$ and $\mathbf{E}(\mathbf{r})$ at each point $\mathbf{r}$ within the dielectric. For homogeneous fluid dielectrics (which are all that we shall be concerned with) the coefficient of proportionality is taken to be a position-independent scalar, which for historical reasons is denoted by $(\epsilon-1) / 4 \pi$. The "dielectric constant" $\epsilon$ is supposed to be a constant of the sample material, dependent only on its temperature and density. Thus, one writes

$$
\mathbf{P}(\mathbf{r})=(\epsilon-1) \mathbf{E}(\mathbf{r}) / 4 \pi \quad(\mathbf{r} \text { in } V) .
$$

Equation (3) has become so canonical and well established that it is necessary to emphasize the following two points, which are sometimes lost sight of: (a) Equation (3) is not in any way to be confused with the macroscopic Maxwell equations, of which it is logically independent. It is rather to be regarded as an auxiliary relation which permits these equations to be solved for $\mathbf{P}(\mathbf{r})$ and $E(\mathbf{r})$, the quantities of interest. (b) Equation (3) cannot simply be regarded as the definition of $\epsilon$, because it is by no means clear that $\mathbf{P}(\mathbf{r})$ and $\mathbf{E}(\mathbf{r})$ (which we have seen are both uniquelydefined physical quantities) in fact bear a constant local proportionality to each other. If they do not, then there exists no constant $\epsilon$ such that Eq. (3) is true, and the dielectric constant is not well defined.

It is clear, then, that Eq. (3) embodies an assump- tion or assertion about the nature of physical reality, and it is appropriate to inquire to what degree of approximation it is correct. Equation (3) has usually been accorded the status of a macroscopic phenomenological relation, the use of which is justified by the fact that its predictions agree with experiment. In our opinion, this point of view is of uncertain soundness in the present case, for there seems to be little direct quantitative experimental evidence in favor of Eq. (3). Most dielectric experiments, such as the usual measurements of the capacitance of a dielectric-filled capacitor, are rather insensitive to the detailed position-dependence of $\mathbf{P}(\mathbf{r})$ [they typically measure a weighted volume average of $\mathbf{P}(\mathbf{r})$ over the sample volume] and are hence insensitive to the precise validity of Eq. (3) as well. There is no question that at least the qualitative features of dielectric polarization are well represented by Eq. (3) ; the question is rather whether Eq. (3) is adequate to predict the detailed position-dependence of $\mathbf{P}(\mathbf{r})$ and $\mathbf{E}(\mathbf{r})$.

The "proper" way to investigate the validity of Eq. (3) is of course by means of a molecular theory. ${ }^{3}$ Such an approach would (ideally) reveal the conditions under which Eq. (3) is valid, and would in the process automatically yield a molecular expression for the parameter $\epsilon$. The present theory provides, for the case of polar fluids, a first step in this direction. Our theory differs from earlier work in that the validity of $\mathrm{Eq}$. (3) is not assumed (nor is any assumption whatever made about the form of the constitutive relation). In contrast, previous theories of dielectric polarization in fluids ${ }^{1,4-12}$ have without apparent exception adopted the assumption (frequently implicit) that Eq. (3) is valid, and have devoted their efforts solely to obtaining theoretical expressions for the dielectric constant on the basis of this assumption. Such a procedure is logically unsatisfactory, and runs the risk that a consistent level of approximation is not maintained. It would clearly be preferable to obtain one's molecular expression for $\epsilon$ as a by-product of a molecular justification of the relation (3) in which this parameter appears. As things stand now, the question of whether the dielectric constant is in fact well defined must be answered on a molecular basis before quantitative significance can safely be attributed to the various extant molecular expressions for it.

In this article we shall be concerned with these considerations only as they apply to polar fluids. It is important in dielectric theory to distinguish clearly in one's mind between the behavior of polar and nonpolar dielectrics, for the physical processes responsible for dielectric polarization are rather different in the two cases. In the former case, the process of primary importance is the lining-up of the permanent molecular dipole moments in the field, while in the latter case it is the production of induced molecular moments by the field. A closely related phenomenon in which induced molecular moments are of primary importance is the 
propagation of light through a material medium. (Permanent moments do not contribute to the polarization at optical frequencies because the field changes too rapidly for them to follow.) In fact, existing molecular theories of light propagation and the refractive inde $x^{13}$ contain features which are probably relevant to the question of the validity of Eq. (3) for nonpolar dielectrics. The relevance of these developments to this question for polar fluids, however, is somewhat more doubtful, since in polar fluids the effects of induced moments are secondary in importance to those of the permanent moments. Since our concern in this article is solely with polar fluids, we shall not find it advantageous to attempt to adapt or modify these existing theories of light propagation. Our particular interest in polar fluids stems from the long-range nature of the permanent dipole-dipole interaction between their constituent molecules, an interaction which appears to give rise in turn to long-range orientational correlations between molecules in zero applied field. (No analogous effect of any importance is expected to occur in nonpolar fluids, since there the long-range dipolar effects are, for the most part, induced by the applied field and therefore vanish when the field is removed.) The macroscopic consequences of these long-range effects on the molecular level, and the manner in which these consequences become realized, are questions of considerable interest even outside the framework of dielectric theory. These questions, about which very little is presently known, provide a special incentive to the study of polar dielectrics in particular.

We must also mention that a certain amount is known about the form of the constitutive relation for certain model lattice systems. The best-known and earliest such result is that of Lorent $z^{14}$ concerning a rigid cubic lattice of isotropically-polarizable particles. More recent developments ${ }^{15}$ have occurred which are of probable relevance to the question at hand for solid dielectrics, but this work is of doubtful relevance to fluids. The reason is that the atoms or molecules remain localized in a solid, but in a fluid they become blurred into a continuum by the statistical averaging. This is not a trivial point, for it means that the dielectric theory of solids is largely free from the conceptual problem one encounters in trying to regard a dielectric continuum as some kind of limiting case of a discrete distribution of dipoles. ${ }^{2}$ Conversely, it means that one unfortunately cannot expect to gain much insight into the conditions under which Eq. (3) is valid for fluids by considering rigid lattices.

We conclude the Introduction with an outline of the organization of the paper. In Sec. II we describe the molecular model under consideration, which basically consists of a rigid unpolarizable molecule with a permanent dipole moment. In Sec. III we give the derivation of our basic equation for $\mathbf{P}(\mathbf{r})$, which expresses $\mathbf{P}(\mathbf{r})$ in terms of $\mathbf{E}_{0}(\mathbf{r})$ as a superposition integral. This derivation involves no approximation except the restriction to responses linear in the applied field, and no assumptions except that in zero applied field $\mathbf{P}(\mathbf{r})$ vanishes and the density is uniform. The derivation is very simple and is of a type which has become exceedingly common in physics; it consists simply in expanding the field-dependent part of the Boltzmann weighting factor and retaining only the linear term. It is therefore procedurally isomorphic to the usual derivations of molecular expressions for the dielectric constant. The principal difference is in the viewpoint - we do not wish to assume that the dielectric constant is well defined, so this assumption, which is commonly built into dielectric theories at the beginning, is omitted.

In Sec. IV we discuss the probable existence of longrange orientational correlations in polar fluids. These correlations constitute a serious complication (in addition to the usual problem that statistical averages involving large numbers of molecules are inherently intractable), and prevent one from using the rigorous result of Sec. III to immediately investigate the validity of Eq. (3) for dense fluids. However, it is readily possible to expand the relevant quantities in powers of the density and investigate the validity of Eq. (3) term by term at low density. In Sec. $V$ we carry this program out to second order in the density, and find that at least to this order Eq. (3) is rigorously valid for our molecular model. To our knowledge, this result constitutes the first theoretical indication that Eq. (3) is other than qualitatively valid for polar fluids under any circumstances. In deriving this result, we obtain some valuable insight into the connection between long-range dipolar effects on the macroscopic and molecular levels. We also obtain automatically expressions for the first and second "dielectric virial coeffcients"; these are found to agree with expressions obtained by previous workers under the assumption that Eq. (3) is valid. Finally, in Sec. VI we give a brief discussion of our results.

\section{THE MOLECULAR MODEL}

The molecular model which we shall adopt will be called the "rigid-dipole" model, and is defined as follows: (a) The molecules are, for simplicity, axially symmetric. (b) The molecules are completely rigid; they do not vibrate and they are not polarizable. (c) Each molecule possesses a permanent dipole moment of magnitude $\mu_{0}$ and direction along the symmetry axis of the molecule. Since the molecules are not polarizable, the total dipole moment of each molecule is just its permanent moment, and is not affected by external fields or by interaction with other molecules. (d) The potential energy $U_{0}\left(\mathbf{Q}^{N}\right)$ of the $N$-molecule system in zero applied field can be written as the sum of pair potentials

$$
U_{0}\left(\mathbf{Q}^{N}\right)=\sum_{i<j} u\left(\mathbf{Q}_{i}, \mathbf{Q}_{j}\right),
$$

where $u\left(\mathbf{Q}_{i}, \mathbf{Q}_{j}\right)$ is the intermolecular pair potential, 
$Q_{i}$ represents the position $R_{i}$ and the orientation $\boldsymbol{\omega}_{i}$ of molecule $i$, and $\mathbf{Q}^{N} \equiv\left(\mathrm{Q}_{1}, \mathrm{Q}_{2}, \cdots, \mathrm{Q}_{N}\right)$. The orientation $\boldsymbol{\omega}_{i}$ may be specified by the usual azimuthal and polar angles $\left(\theta_{i}, \phi_{i}\right)$ defining the direction of the permanent moment of molecule $i$ with respect to some arbitrary laboratory reference frame.

The intermolecular pair potential $u\left(Q_{1}, Q_{2}\right)$ is the sum of the usual permanent dipole-dipole interaction energy and a short-range interaction energy. The shortrange energy may be left unspecified except for the following two conditions: (1) It tends to positive infinity as the intermolecular separation $\left|\mathbf{R}_{\mathbf{1}}-\mathbf{R}_{\mathbf{2}}\right|$ tends to zero, becoming much larger than $\beta^{-1} \equiv k T$ when $\left|\mathbf{R}_{\mathbf{1}}-\mathbf{R}_{\mathbf{2}}\right|<r_{0}$, where $r_{0} \sim 10^{-8} \mathrm{~cm}$ sets the general order of magnitude of this "hard-core" repulsion. (2) It goes to zero with increasing $\left|\mathbf{R}_{\mathbf{1}}-\mathbf{R}_{\mathbf{2}}\right|$ faster than $\left|\mathbf{R}_{1}-\mathbf{R}_{\mathbf{2}}\right|^{-3}$, becoming negligible compared to both $k T$ and the dipole-dipole energy when $\left|\mathbf{R}_{1}-\mathbf{R}_{2}\right|>\sigma$, where $\sigma$ is a distance of molecular magnitude which may be many molecular diameters but which must be small macroscopically (i.e., $\sigma \lesssim 10^{-3} \mathrm{~cm}$ ). In addition to the hard-core repulsion, the short-range part of the potential may be thought of as being due to the existence of molecular multipole moments of higher order than the dipole, or to other causes.

Since the magnitude of each molecular moment is constant, it is convenient to introduce unit vectors collinear with the moments. The moment of molecule $k, \boldsymbol{\mu}_{k}$, can then be written as

$$
\boldsymbol{\mu}_{k}\left(\boldsymbol{\omega}_{k}\right)=\mu_{0} \mathbf{e}\left(\boldsymbol{\omega}_{k}\right),
$$

where $\mathbf{e}(\boldsymbol{\omega})$ is the unit vector with direction $\boldsymbol{\omega}$,

$$
\mathbf{e}(\boldsymbol{\omega})=\sin \theta \cos \phi \mathbf{e}_{x}+\sin \theta \sin \phi \mathbf{e}_{y}+\cos \theta \mathbf{e}_{z} ;
$$

$\mathbf{e}_{\alpha}(\alpha=x, y, z)$ is the unit vector along the $\alpha$ direction of the laboratory frame.

Since we can neglect the short-range part of the pair potential in comparison to the dipole-dipole interaction if the intermolecular separation exceeds $\sigma$, we can write

$$
\begin{array}{r}
u\left(\mathbf{Q}_{1}, \mathbf{Q}_{2}\right)=-\mu_{0}^{2} \mathbf{T}\left(\mathbf{R}_{1}-\mathbf{R}_{2}\right): \mathbf{e}\left(\boldsymbol{\omega}_{1}\right) \mathbf{e}\left(\boldsymbol{\omega}_{2}\right) \\
\text { if }\left|\mathbf{R}_{\mathbf{1}}-\mathbf{R}_{\mathbf{2}}\right|>\sigma .
\end{array}
$$

Having defined the rigid-dipole model, we must say something about the degree to which it represents physical reality. One might at first expect this model to be a good approximation in the case of real highly polar fluids. All real molecules are polarizable, however, and there is good reason to believe that the effects of molecular polarizability are considerable even in highly polar fluids., ${ }^{2,6,12,16}$ The rigid-dipole model must therefore be regarded as an inadequate basis for the quantitative interpretation of the behavior of real polar dielectrics. It is adopted here because it is expected to exhibit qualitatively the essential features of dielectric polarization in real polar substances, while avoiding the truly vast increase in complexity which the addition of molecular polarizability would intro- duce into the theory., ${ }^{2,11,12}$ The effects of molecular polarizability, although not negligible, are certainly secondary in importance to the effects of the permanent dipole moments, and to take them into account would complicate the theory to a much greater extent than their importance warrants. The consequences of the long-range nature of the dipole-dipole potential are our main concern here, and it is advisable to approach their study in the absence of serious but nonessential complications which could tend to obscure the fundamental processes involved.

\section{DERIVATION OF THE FUNDAMENTAL EQUATION FOR P(r)}

We will now derive our basic molecular expression for the polarization $\mathbf{P}(\mathbf{r})$. Before beginning, however, we emphasize that we are still considering the physical situation described in the Introduction: a finite macroscopic sample of fluid dielectric consisting of $N$ identical rigid-dipole molecules in a volume $V$, of definite shape $S$, at absolute temperature $T$. The sample is suspended in vacuum. We want to calculate the polarization $\mathbf{P}(\mathbf{r})$ produced in this sample by a weak external field $\mathbf{E}_{0}(\mathbf{r})$.

The microscopic dynamical variable which corresponds to the macroscopic dipole moment per unit volume $\mathbf{P}(\mathbf{r})$ is

$$
\mathbf{P}\left(\mathbf{Q}^{N} ; \mathbf{r}\right)=\sum_{k=1}^{N} \boldsymbol{\mu}_{k} \delta\left(\mathbf{r}-\mathbf{R}_{k}\right)=\mu_{0} \sum_{k=1}^{N} \mathbf{e}\left(\boldsymbol{\omega}_{k}\right) \delta\left(\mathbf{r}-\mathbf{R}_{k}\right) .
$$

The macroscopic polarization $\mathbf{P}(\mathbf{r})$ is obtained by averaging $\mathbf{P}\left(\mathbf{Q}^{N} ; \mathbf{r}\right)$ over $\mathbf{Q}^{N}$ in the presence of the external field

$$
\begin{aligned}
\mathbf{P}(\mathbf{r})=\left\langle\mathbf{P}\left(\mathbf{Q}^{N} ; \mathbf{r}\right)\right\rangle_{\boldsymbol{E}} & =\mu_{0} \sum_{k=1}^{N}\left\langle\mathbf{e}\left(\boldsymbol{\omega}_{k}\right) \delta\left(\mathbf{r}-\mathbf{R}_{k}\right)\right\rangle_{E} \\
& =N \mu_{0}\left\langle\mathbf{e}\left(\boldsymbol{\omega}_{1}\right) \delta\left(\mathbf{r}-\mathbf{R}_{1}\right)\right\rangle_{E},
\end{aligned}
$$

where we have made use of the fact that all the molecules are identical to focus attention on the representative molecule 1 . The notation $\langle\cdots\rangle_{E}$ denotes a canonical statistical-mechanical average over all $\mathrm{Q}^{N}$ (i.e., over all positions and orientations of the molecules), weighted by the Boltzmann factor appropriate to equilibrium in the presence of $\mathbf{E}_{0}(\mathbf{r})$. That is,

$$
\left\langle A\left(\mathbf{Q}^{N}\right)\right\rangle_{E}=\frac{\int d \mathbf{Q}^{N} A\left(\mathbf{Q}^{N}\right) \exp \left\{-\beta U_{E}\left(\mathbf{Q}^{N}\right)\right\}}{\int d \mathbf{Q}^{N} \exp \left\{-\beta U_{E}\left(\mathbf{Q}^{N}\right)\right\}},
$$

where $U_{E}\left(\mathbf{Q}^{N}\right)$ is the potential energy of the sample in a given configuration $\mathrm{Q}^{N}$ and in the presence of $\mathrm{E}_{0}(\mathbf{r})$, and $A\left(\mathbf{Q}^{N}\right)$ is an arbitrary observable. The volume element in configuration space $d \mathbf{Q}^{N}$ is to be interpreted in the following way:

$$
\begin{aligned}
d \mathbf{Q}^{N} & =\prod_{i=1}^{N} d \mathbf{Q}_{i}, \\
d \mathbf{Q}_{i} & =d^{3} \mathbf{R}_{i} d \boldsymbol{\omega}_{i}, \\
d \boldsymbol{\omega}_{i} & =\sin \theta_{i} d \theta_{i} d \boldsymbol{\phi}_{i},
\end{aligned}
$$


$\theta_{i}$ varies between 0 and $\pi$ and $\phi_{i}$ varies between 0 and $2 \pi$. The integrations over molecular positions $\mathbf{R}_{i}$ are of course to be extended only over the volume $V$.

We shall assume that the quantity $\mathbf{P}(\mathbf{r})$ given by Eq. (9) has the property of being slowly-varying in a molecular sense, by virtue of the averaging over molecular configurations. This assumption is exceedingly reasonable for a fluid, although of course quite false for a solid.

The potential energy in the presence of the external field, $U_{E}\left(\mathbf{Q}^{N}\right)$, is just the sum of the potential energy in zero field, $U_{0}\left(\mathbf{Q}^{N}\right)$, and the energy of interaction between the sample in configuration $\mathbf{Q}^{N}$ and the field $\mathbf{E}_{0}(\mathbf{r})$. The latter is given by

$$
-\sum_{k} \boldsymbol{\mu}_{k} \cdot \mathbf{E}_{0}\left(\mathbf{R}_{k}\right)=-\mu_{0} \sum_{k} \mathbf{e}\left(\boldsymbol{\omega}_{k}\right) \cdot \mathbf{E}_{0}\left(\mathbf{R}_{k}\right)
$$

since the field $E_{0}(r)$ is essentially uniform over any given molecule (i.e., the slow variation of $\mathbf{E}_{0}(\mathbf{r})$ with $\mathbf{r}$ ensures that only the dipole moment of each molecule, and no higher multipole, interacts with it). Therefore,

$$
U_{E}\left(\mathbf{Q}^{N}\right)=U_{0}\left(\mathbf{Q}^{N}\right)-\mu_{0} \sum_{k} \mathbf{e}\left(\boldsymbol{\omega}_{k}\right) \cdot \mathbf{E}_{0}\left(\mathbf{R}_{k}\right)
$$

Substituting Eq. (13) into Eq. (10) and dividing both numerator and denominator by $\int d \mathbf{Q}^{N} \exp \left\{-\beta U_{0}\left(\mathbf{Q}^{N}\right)\right\}$ yields

$$
\left\langle A\left(\mathbf{Q}^{N}\right)\right\rangle_{E}=\frac{\left\langle A\left(\mathbf{Q}^{N}\right) \exp \left\{\beta \mu_{0} \sum_{k} \mathbf{e}\left(\boldsymbol{\omega}_{k}\right) \cdot \mathbf{E}_{0}\left(\mathbf{R}_{k}\right)\right\}\right\rangle_{0}}{\left\langle\exp \left\{\beta \mu_{0} \sum_{k} \mathbf{e}\left(\boldsymbol{\omega}_{k}\right) \cdot \mathbf{E}_{0}\left(\mathbf{R}_{k}\right)\right\}\right\rangle_{0}},
$$

where the notation $\langle\cdots\rangle_{0}$ denotes an average taken in zero applied field

$$
\left\langle A\left(\mathbf{Q}^{N}\right)\right\rangle_{0}=\frac{\int d \mathbf{Q}^{N} A\left(\mathbf{Q}^{N}\right) \exp \left\{-\beta U_{0}\left(\mathbf{Q}^{N}\right)\right\}}{\int d \mathbf{Q}^{N} \exp \left\{-\beta U_{0}\left(\mathbf{Q}^{N}\right)\right\}} .
$$

Equation (14) constitutes the formal reduction of the problem of evaluating an average in the presence of the field to that of evaluating other averages taken in the unperturbed system (zero applied field).

It is known experimentally that a dielectric in zero applied field produces no field of its own. ${ }^{17}$ Therefore $\mathbf{P}(\mathbf{r})$ is zero in zero applied field, which from Eq. (9) implies that

$$
\left\langle\mathbf{e}\left(\boldsymbol{\omega}_{1}\right) \delta\left(\mathbf{r}-\mathbf{R}_{1}\right)\right\rangle_{0}=0 .
$$

Replacing $A\left(\mathbf{Q}^{N}\right)$ by $\mathbf{e}\left(\boldsymbol{\omega}_{1}\right) \delta\left(\mathbf{r}-\mathbf{R}_{1}\right)$ in Eq. (14), expanding the result to first order in $\mathrm{E}_{0}(\mathbf{r})$, making use of Eq. (16), and substituting into Eq. (9), we obtain

$\mathbf{P}(\mathbf{r})=N \mu_{0}^{2} \beta\left\langle\mathbf{e}\left(\boldsymbol{\omega}_{1}\right) \delta\left(\mathbf{r}-\mathbf{R}_{1}\right) \sum_{k=1}^{N} \mathbf{e}\left(\boldsymbol{\omega}_{k}\right) \cdot \mathbf{E}_{0}\left(\mathbf{R}_{k}\right)\right\rangle_{0}$.

By introducing another Dirac delta function, Eq. (17) can be written in the form

$$
\mathbf{P}(\mathbf{r})=\int d^{3} \mathbf{r}^{\prime} \mathbf{K}\left(\mathbf{r}, \mathbf{r}^{\prime}\right) \cdot \mathbf{E}_{\mathbf{0}}\left(\mathbf{r}^{\prime}\right),
$$

where

$$
\begin{aligned}
\mathbf{K}\left(\mathbf{r}, \mathbf{r}^{\prime}\right) & =N \mu_{0}^{2} \beta\left\langle\mathbf{e}\left(\boldsymbol{\omega}_{1}\right) \delta\left(\mathbf{r}-\mathbf{R}_{1}\right) \sum_{k=1}^{N} \mathbf{e}\left(\boldsymbol{\omega}_{k}\right) \delta\left(\mathbf{r}^{\prime}-\mathbf{R}_{k}\right)\right\rangle_{0} \\
& =N \mu_{0}^{2} \beta\left[\left\langle\mathbf{e}\left(\boldsymbol{\omega}_{1}\right) \mathbf{e}\left(\boldsymbol{\omega}_{1}\right) \delta\left(\mathbf{r}-\mathbf{R}_{1}\right)\right\rangle_{0} \delta\left(\mathbf{r}-\mathbf{r}^{\prime}\right)\right. \\
+ & \left.(N-1)\left\langle\mathbf{e}\left(\boldsymbol{\omega}_{1}\right) \mathbf{e}\left(\boldsymbol{\omega}_{2}\right) \delta\left(\mathbf{r}-\mathbf{R}_{1}\right) \delta\left(\mathbf{r}^{\prime}-\mathbf{R}_{2}\right)\right\rangle_{0}\right] .
\end{aligned}
$$

It is understood that the integration over $\mathbf{r}^{\prime}$ in Eq. (18) is extended only over the volume $V$ occupied by the sample. This will also be understood, unless otherwise stated, in all subsequent expressions in this article.

It is desirable to put $K\left(\mathbf{r}, \mathbf{r}^{\prime}\right)$ into a better form than that of Eq. (19). To do so, we first notice that Eq. (16) implies an isotropic distribution of directions of $\mathbf{e}\left(\omega_{1}\right)$ for fixed $\mathbf{R}_{1}$ in zero field. Therefore $\left\langle\mathbf{e}\left(\boldsymbol{\omega}_{1}\right) \mathbf{e}\left(\boldsymbol{\omega}_{1}\right) \delta\left(\mathbf{r}-\mathbf{R}_{1}\right)\right\rangle_{0}$ must be proportional to $\mathbf{U}$, the unit dyadic

$$
\left\langle\mathbf{e}\left(\boldsymbol{\omega}_{1}\right) \mathbf{e}\left(\boldsymbol{\omega}_{1}\right) \delta\left(\mathbf{r}-\mathbf{R}_{1}\right)\right\rangle_{0}=c(\mathbf{r}) \mathbf{U} .
$$

The coefficient of proportionality $c(\mathbf{r})$ can be obtained by double-dotting $U$ into both sides of Eq. (20) and using the fact that $U: U=3$ :

$$
\begin{aligned}
c(\mathbf{r})=(1 / 3)\left\langle\mathbf{e}\left(\boldsymbol{\omega}_{1}\right) \mathbf{e}\left(\boldsymbol{\omega}_{1}\right) \delta\left(\mathbf{r}-\mathbf{R}_{1}\right)\right\rangle_{0}: \mathrm{U} \\
=(1 / 3)\left\langle\delta\left(\mathbf{r}-\mathbf{R}_{1}\right)\right\rangle_{0} .
\end{aligned}
$$

But $N\left\langle\delta\left(\mathbf{r}-\mathbf{R}_{1}\right)\right\rangle_{0}$ is just the number density (number of molecules per unit volume) at the point $\mathbf{r}$ in zero field. We shall assume that the number density in zero field is uniform and equal to the constant value $\rho$, independent of $\mathbf{r}$. Then $c(\mathbf{r})=\rho / 3 N$ and Eq. (19) becomes

$$
\begin{aligned}
& \mathbf{K}\left(\mathbf{r}, \mathbf{r}^{\prime}\right)=(1 / 3) \rho \mu_{0}^{2} \beta \mathbf{U} \delta\left(\mathbf{r}-\mathbf{r}^{\prime}\right) \\
& +N(N-1) \mu_{0}^{2} \beta\left\langle\mathbf{e}\left(\boldsymbol{\omega}_{1}\right) \mathbf{e}\left(\boldsymbol{\omega}_{2}\right) \delta\left(\mathbf{r}-\mathbf{R}_{1}\right) \delta\left(\mathbf{r}^{\prime}-\mathbf{R}_{2}\right)\right\rangle_{0} .
\end{aligned}
$$

Let us define the two-molecule orientation-dependent generic distribution function $\rho^{(2)}$ in the usual way; i.e.,

$$
\begin{aligned}
\rho^{(2)}\left(\mathbf{Q}_{1}, \mathbf{Q}_{2}\right) & =\rho^{(2)}\left(\mathbf{R}_{1}, \boldsymbol{\omega}_{1} ; \mathbf{R}_{2}, \boldsymbol{\omega}_{2}\right) \\
& =N(N-1) \frac{\int d \mathbf{Q}^{N-2} \exp \left\{-\beta U_{0}\left(\mathbf{Q}^{N}\right)\right\}}{\int d \mathbf{Q}^{N} \exp \left\{-\beta U_{0}\left(\mathbf{Q}^{N}\right)\right\}},
\end{aligned}
$$

where

$$
d \mathbf{Q}^{N-2}=\prod_{i=3}^{N} d \mathbf{Q}_{i} .
$$

It is understood that Eq. (23) is to be used only if both $\mathbf{R}_{1}$ and $\mathbf{R}_{2}$ are in the sample volume $V$, and that $\rho^{(2)}=0$ otherwise. In terms of $\rho^{(2)}$, Eq. (22) becomes

$$
\begin{aligned}
\mathbf{K}\left(\mathbf{r}, \mathbf{r}^{\prime}\right) & =\mu_{0}^{2} \beta\left[(1 / 3) \rho \mathbf{U} \delta\left(\mathbf{r}-\mathbf{r}^{\prime}\right)\right. \\
& \left.+\int d \boldsymbol{\omega}_{1} d \boldsymbol{\omega}_{2} \boldsymbol{\rho}^{(2)}\left(\mathbf{r}, \boldsymbol{\omega}_{1} ; \mathbf{r}^{\prime}, \boldsymbol{\omega}_{2}\right) \mathbf{e}\left(\boldsymbol{\omega}_{1}\right) \mathbf{e}\left(\boldsymbol{\omega}_{2}\right)\right]
\end{aligned}
$$

which is our final form for $K\left(\mathbf{r}, \mathbf{r}^{\prime}\right)$.

Equations (18) and (24) constitute the basic result of our theory. Equation (18) expresses the polarization $\mathbf{P}(\mathbf{r})$ at the point $\mathbf{r}$ as a linear superposition of the 
effects due to $\mathbf{E}_{0}\left(\mathbf{r}^{\prime}\right)$ at all points $\mathbf{r}^{\prime}$ in the sample volume. The dyadic kernel $\mathbf{K}\left(\mathbf{r}, \mathbf{r}^{\prime}\right)$ which governs this superposition may be regarded as the fundamental quantity of dielectric theory in much the same sense that the response function is the fundamental quantity of linear response theory..$^{18}$ All the statistical mechanics, and consequently all the physics of the problem [except for the linearity assumption, which is built into Eq. (18) ], is contained in $K\left(r, r^{\prime}\right)$. It is this quantity which determines, through Eq. (18), the way in which a particular sample (i.e., having a particular volume and shape) of a particular dielectric substance behaves when subjected to an arbitrary external field $\mathbf{E}_{0}(\mathbf{r})$. There is no reason to suppose that $K\left(r, r^{\prime}\right)$ does not depend, in general, upon the volume and shape of the sample as well as upon the material of which it is composed.

The problem of computing $\mathbf{P}(\mathbf{r})$ for a system of rigid-dipole molecules has therefore been reduced to the problem of calculating $\mathbf{K}\left(\mathbf{r}, \mathbf{r}^{\prime}\right)$, and this latter problem reduces because of $\mathrm{Eq}$. (24) to that of evaluating the pair distribution function of the unperturbed fluid. Since $\int d \boldsymbol{\omega} \mathbf{e}(\boldsymbol{\omega})=0$, we see from Eq. (24) that $\mathrm{K}\left(\mathbf{r}, \mathbf{r}^{\prime}\right)$ will be nonzero (for $\left.\mathbf{r} \neq \mathbf{r}^{\prime}\right)$ only if there exists an angular correlation between two representative molecules located at $\mathbf{r}$ and $\mathbf{r}^{\prime}$ in the unperturbed fluid. This implies, through Eq. (18), that the external field at the point $\mathbf{r}^{\prime}$ will produce a contribution to the polarization at the point $\mathbf{r}\left(\mathbf{r} \neq \mathbf{r}^{\prime}\right)$ only if there exists an angular correlation in zero applied field between two molecules located at $\mathbf{r}$ and $\mathbf{r}^{\prime}$. The reciprocity or symmetry which one feels must exist between the points $\mathbf{r}$ and $\mathbf{r}^{\prime}$ is expressed by the symmetry relation

$$
\mathbf{K}\left(\mathbf{r}, \mathbf{r}^{\prime}\right)=\mathbf{K}^{T}\left(\mathbf{r}^{\prime}, \mathbf{r}\right),
$$

where the superscript $T$ denotes the transpose. Equation (25) can be verified easily from Eq. (24).

We emphasize again that the only assumptions (besides linearity) we have made are that in zero field the polarization $\mathbf{P}(\mathbf{r})$ is zero and the density is uniform. The volume and shape of the sample, as well as the functional form of $\mathbf{E}_{0}(\mathbf{r})$, have been left arbitrary.

\section{LONG-RANGE ANGULAR CORRELATIONS}

In principle, the results of the preceding section [embodied in Eqs. (18) and (24)] can be used to investigate whether the dielectric constant is well defined; i.e., whether there exists a constant $\epsilon$ such that Eq. (3) is true. One might think that this could be done simply by solving Eq. (1) for $\mathbf{E}_{0}(\mathbf{r})$ and substituting the result into Eq. (18) to obtain a rigorous relation between $\mathbf{P}(\mathbf{r})$ and $E(\mathbf{r})$, which could then be compared with Eq. (3). When this is done, however, the result bears no apparent resemblance to $\mathrm{Eq}$. (3), and it becomes apparent that one must know something about the properties of $K\left(r, r^{\prime}\right)$ in order to make further progress. This in turn requires, because of Eq. (24), knowledge about the pair distribution function $\rho^{(2)}$. The rigorous evaluation of this quantity is unfortunately not feasible for a dense fluid, and methods for approximating it are not well developed. Moreover, in the case of polar fluids it is probable that the pair distribution function contains long-range orientational correlations (i.e., angular correlations between molecules separated by macroscopic distances) in addition to the usual short-range correlations. Detailed knowledge about the nature of these long-range correlations is essential if one is to investigate the validity of $\mathrm{Eq}$. (3) by the procedure outlined above.

The probable existence of long-range orientational correlations in polar fluids can be inferred in several ways, of which we briefly mention three:

(a) Any long-range orientational correlations present in polar fluids must of course be basically due to the long-range character of the dipole-dipole part of the intermolecular potential..$^{19}$ The pair distribution function is obtained by fixing the positions and orientations of molecules 1 and 2 and averaging over the positions and orientations of the remaining $N-2$ molecules. This averaging process creates a "statistical continuum" in which molecules 1 and 2 may be considered to be embedded, and their mutual dipolar interaction, while effectively modified by the polarization they create in this statistical continuum, will very likely still possess the long-range $r^{-3}$ radial dependence and the angular dependence characteristic of the dipolar interaction. It is true that in many contexts the angular dependence of the dipole-dipole potential, which averages over an isotropic angular distribution to zero, renders the dipole-dipole potential effectively short-ranged. ${ }^{2}$ However, in calculating $\boldsymbol{\rho}^{(2)}\left(\mathbf{R}_{\mathbf{1}}, \boldsymbol{\omega}_{1} ; \mathbf{R}_{\mathbf{2}}, \boldsymbol{\omega}_{2}\right)$, the angles represented by $\boldsymbol{\omega}_{1}$ and $\boldsymbol{\omega}_{2}$ are held fixed and are not averaged over, so this angular-averaging effect does not come into play.

(b) If one assumes the validity of Eq. (3), as has customarily been done in dielectric theory, one finds that the molecular averages which appear in the resulting expression for $\epsilon$ are strongly dependent upon the shape of the sample. ${ }^{2 a}$ For a rigid-dipole fluid, these averages can be expressed in terms of the pair distribution function, and it is difficult to imagine how the shape-dependence could arise if this quantity were entirely short-ranged in character.

(c) We can also infer the existence of long-range angular correlations within the framework of the present theory. If the correlations were entirely shortranged, Eqs. (18) and (24) imply that the polarization $\mathbf{P}(\mathbf{r})$ at the point $\mathbf{r}$ would depend only upon the external field at points in the molecular neighborhood of $\mathbf{r}$. But if the external field were very strong at points, say, $1 \mathrm{~cm}$ or more from $r$ it would produce a large polarization at these points, and the field produced by this polarization would act to further polarize the dielectric at $\mathbf{r}$. It is thus unreasonable to expect a local relation between $\mathbf{P}(\mathbf{r})$ and $\mathrm{E}_{0}(\mathbf{r})$, and because 
of Eqs. (18) and (24) a nonlocal relation between $\mathbf{P}(\mathbf{r})$ and $\mathbf{E}_{0}(\mathbf{r})$ implies angular correlations of a nonlocal (i.e., long-range) nature.

We therefore see that there is good reason to believe that long-range orientational correlations exist in polar liquids even at ordinary temperatures. It is important to realize that the effect of such long-range correlations is enhanced by the fact that they act over a much greater volume than the usual short-range correlations, and that as a consequence very weak long-range correlations can easily exert an effect comparable to that of much stronger short-range correlations. This can easily be seen in the present context by inspection of Eqs. (18) and (24). From Eq. (24) we see that if $\rho^{(2)}$ contains long-range angular correlations then $K\left(r, r^{\prime}\right)$ can differ from zero even if $\left|\mathbf{r}-\mathbf{r}^{\prime}\right|$ is a distance of macroscopic size. Because of the volume integration in Eq. (18), it is therefore clear that the total contribution to $\mathbf{P}(\mathbf{r})$ of very weak long-range correlations could easily be comparable to or greater than that of very strong short-range correlations. It is tempting to speculate that the pair distribution function can be resolved into well-defined short-range and long-range parts, and that the long-range part could be approximated on the basis of suitable macroscopic considerations if only one knew how to do so.

It is worth mentioning that the existence of longrange orientational correlations in polar liquids is not of interest solely in connection with their dielectric behavior, for such correlations could also exert pronounced effects on other observable properties, such as the familiar thermodynamic functions.

In summary, then, the use of our basic results to investigate the validity of Eq. (3) in dense polar fluids is prevented at present by the fact that the pair distribution function for such fluids must be usefully approximated before any progress can be made. In particular, some approximate means of taking into account the effects of the long-range angular correlations must be developed, and at present very little is known about how to deal with this interesting problem. The development of a suitable approximation scheme for the longrange correlations must be assigned the highest priority in future work.

In the case of dilute gases, however, we are fortunately not forced to wait for a fundamental advance in the theory in order to make further progress: We can actually evaluate the pair distribution function at low density by means of a density expansion. In the next section we use this approach to demonstrate that the dielectric constant is in fact well defined at least to second order in the density.

\section{LOW-DENSITY BEHAVIOR}

For convenience we rewrite here Eq. (3), replacing $(\epsilon-1) / 4 \pi$ by $\chi$,

$$
\mathbf{P}(\mathbf{r})=\chi \mathbf{E}(\mathbf{r}) \quad(\mathbf{r} \text { in } V) .
$$

If there exists a constant $\chi$, dependent only on the sample material and its temperature and density, such that Eq. (26) is satisfied, then the dielectric constant is in fact well defined and is equal to

$$
\epsilon=1+4 \pi \chi \text {. }
$$

If no such $\chi$ exists, the dielectric constant is not well defined.

We have seen that it is not at present feasible to investigate the existence of $\chi$ for dense fluids, although for dilute gases we expect to be able to proceed by means of a density expansion. It is of course conceivable that a $\chi$ can be found which satisfies Eq. (26) for sufficiently low densities but not for higher ones. This consideration makes the following definition natural: If there exists a $\chi$ such that

$$
\lim _{\rho \rightarrow 0}\left\{\rho^{-k}[\mathbf{P}(\mathbf{r})-\chi \mathrm{E}(\mathbf{r})]\right\}=0 \quad(\mathbf{r} \text { in } V),
$$

where $k$ is an integer $\geq 0$, then we will say that $\epsilon$ is well defined at least to $k$ th order in the density. We can say "at least" because it is clear that if Eq. (28) is satisfied with $k=m$ it will also be satisfied with $k=m^{\prime}<m$. If $k$ is the largest integer for which a $\chi$ exists such that Eq. (28) holds, then we say that $\epsilon$ is well defined only to $k$ th order in the density. If a $\chi$ can be found such that Eq. (28) holds for all $k$, then $\epsilon$ is well defined to any order in the density.

We can therefore regard $\chi(\rho)$ as an unknown function of $\rho$ and attempt to determine it to a given order $k$ by requiring that it satisfy $\mathrm{Eq}$. (28) for that value of $k$. If we succeed then we will have shown that $\epsilon$ is well defined at least to $k$ th order in the density, and will have obtained in the process an explicit expression for it to that order. If we fail then we know that $\epsilon$ is not well defined to $k$ th order; we might then decide to repeat the investigation using a smaller value of $k$.

It is desirable to dispose of the trivial case $k=0$ at the outset. It is clear that $\mathbf{P}(\mathbf{r}) \rightarrow 0$ and that $\mathbf{E}(\mathbf{r}) \rightarrow \mathbf{E}_{0}(\mathbf{r})$ as $\rho \rightarrow 0$. We therefore see that any $\chi$ which vanishes at $\rho=0$ will satisfy Eq. (28) for the case $k=0$. Having noted this, we can henceforth limit our consideration to functions $\chi(\rho)$ which vanish at $\rho=0$, and to values of $k$ in Eq. (28) which are $\geq 1$.

Since our investigation will proceed by means of a density expansion, let us begin by writing all quantities of interest as power series in the density

$$
\begin{aligned}
& \mathbf{P}(\mathbf{r})=\sum_{k=1}^{\infty} \rho^{k} \mathbf{P}_{k}(\mathbf{r}), \\
& \mathbf{E}(\mathbf{r})=\sum_{k=0}^{\infty} \rho^{k} \mathbf{E}_{k}(\mathbf{r}), \\
& \chi(\boldsymbol{\rho})=\sum_{k=1}^{\infty} \rho^{k} \chi_{k},
\end{aligned}
$$

where we have made use of the known zero-density behavior of these quantities. Note in particular that 
Eq. (29b) contains the fact that $\mathbf{E}(\mathbf{r}) \rightarrow \mathbf{E}_{0}(\mathbf{r})$ as $\rho \rightarrow 0$. Within the framework of a density expansion, the determination of the unknown function $\chi(\rho)$ is done by determining the coefficients $\chi_{k}$ in $\mathrm{Eq} .(29 \mathrm{c})$ in accordance with Eq. (28).

The coefficients $\mathbf{E}_{k}(\mathbf{r})(k \geq 1)$ can immediately be obtained from the $\mathbf{P}_{k}(\mathbf{r})$ by means of the formula

$$
\begin{aligned}
& \mathbf{E}_{k}(\mathbf{r})=-(4 \pi / 3) \mathbf{P}_{k}(\mathbf{r}) \\
& \quad+\lim _{\delta \rightarrow 0} \int_{\left|\mathbf{r}-\mathbf{r}^{\prime}\right|>\delta} d^{3} \mathbf{r}^{\prime} \mathbf{T}\left(\mathbf{r}-\mathbf{r}^{\prime}\right) \cdot \mathbf{P}_{k}\left(\mathbf{r}^{\prime}\right), \quad(k \geq 1),
\end{aligned}
$$

which follows directly from Eq. (1). In order to obtain the $\mathbf{P}_{k}(\mathbf{r})$, we must first express the pair distribution function as a power series in the density. This is done in the Appendix, with the result

$$
\rho^{(2)}\left(\mathbf{R}_{\mathbf{1}}, \boldsymbol{\omega}_{\mathbf{1}} ; \mathbf{R}_{\mathbf{2}}, \boldsymbol{\omega}_{\mathbf{2}}\right)=\sum_{k=2}^{\infty} \boldsymbol{\rho}^{k} \rho_{k}^{(2)}\left(\mathbf{R}_{\mathbf{1}}, \boldsymbol{\omega}_{1} ; \mathbf{R}_{\mathbf{2}}, \boldsymbol{\omega}_{2}\right)
$$

Note that the expansion begins with the second-order term. The coefficients in Eq. (31) can be generated as described in the Appendix. Since we shall carry our investigation only out to second order in the density, we will need an explicit formula only for the coefficient $\rho_{2}{ }^{(2)}$. This formula is found in the Appendix to be

$\rho_{2}{ }^{(2)}\left(\mathbf{R}_{1}, \boldsymbol{\omega}_{1} ; \mathbf{R}_{2}, \boldsymbol{\omega}_{2}\right)=(4 \pi)^{-2} \exp \left\{-\beta u\left(\mathbf{R}_{1}, \boldsymbol{\omega}_{1} ; \mathbf{R}_{2}, \boldsymbol{\omega}_{2}\right)\right\}$,

where $u\left(\mathbf{R}_{\mathbf{1}}, \boldsymbol{\omega}_{1} ; \mathbf{R}_{2}, \boldsymbol{\omega}_{2}\right)$ is the pair potential discussed in Sec. II. Equation (32) is of course applicable only when both $R_{1}$ and $R_{2}$ are in the sample volume $V$, since $\rho_{2}{ }^{(2)}$, like $\rho^{(2)}$ itself, vanishes otherwise. We can now obtain the coefficients $\mathbf{P}_{k}(\mathbf{r})$ by combining Eqs. (18), (24), and (31) and comparing the result with Eq. (29a). We obtain

$$
\begin{array}{r}
\mathbf{P}_{\mathbf{1}}(\mathbf{r})=(1 / 3) \mu_{0}^{2} \beta \mathbf{E}_{0}(\mathbf{r}) \\
\mathbf{P}_{k}(\mathbf{r})=\mu_{0}^{2} \beta \int d^{3} \mathbf{r}^{\prime} \int d \boldsymbol{\omega}_{1} d \boldsymbol{\omega}_{2} \rho_{k}^{(2)}\left(\mathbf{r}, \boldsymbol{\omega}_{1} ; \mathbf{r}^{\prime}, \boldsymbol{\omega}_{2}\right) \mathbf{e}\left(\boldsymbol{\omega}_{\mathbf{1}}\right) \mathbf{e}\left(\boldsymbol{\omega}_{2}\right) \\
\cdot \mathbf{E}_{0}\left(\mathbf{r}^{\prime}\right),
\end{array}
$$

with $k \geq 2$ in Eq. (33b). For the case $k=2$, Eq. (32) implies

$$
\begin{aligned}
& \mathbf{P}_{2}(\mathbf{r})=(4 \pi)^{-2} \mu_{0}^{2} \beta \int d^{3} \mathbf{r}^{\prime} \int d \boldsymbol{\omega}_{1} d \boldsymbol{\omega}_{2} \\
& \quad \times \exp \left\{-\beta u\left(\mathbf{r}, \boldsymbol{\omega}_{1} ; \mathbf{r}^{\prime}, \boldsymbol{\omega}_{2}\right)\right\} \mathbf{e}\left(\boldsymbol{\omega}_{1}\right) \mathbf{e}\left(\boldsymbol{\omega}_{2}\right) \cdot \mathbf{E}_{0}\left(\mathbf{r}^{\prime}\right) .
\end{aligned}
$$

We remind the reader that the integrals over $\mathbf{r}^{\prime}$ in Eqs. (33) are to be extended only over the volume $V$ occupied by the sample. Furthermore, it is clear that these equations are applicable only when $\mathbf{r}$ is in $V$, since the $\mathbf{P}_{k}(\mathbf{r})$ vanish otherwise.

We therefore see that the coefficients $\mathbf{P}_{k}(\mathbf{r})$ and $\mathbf{E}_{k}(\mathbf{r})$, like their parent quantities $\mathbf{P}(\mathbf{r})$ and $\mathbf{E}(\mathbf{r})$, are well defined quantities [given by Eqs. (30) and (33)] regardless of whether or not the dielectric constant is well defined to any particular order. The quantities $\chi_{k}$ are as yet undetermined, and the question we want to answer is to what extent they can be determined in accordance with Eq. (28). To investigate this question, it is desirable to reexpress Eq. (28) in terms of the various coefficients appearing in Eqs. (29). By substituting Eqs. (29) into Eq. (28) and collecting together the coefficients of equal powers of $\rho$, one can show without difficulty that Eq. (28) (with $k \geq 1$ ) is equivalent to the following set of $k$ equations,

$$
\mathbf{P}_{j}(\mathbf{r})=\sum_{m=1}^{j} \chi_{m} \mathbf{E}_{j-m}(\mathbf{r}) \quad(j=1,2, \cdots, k) .
$$

If $k$ constants $\chi_{1}, \chi_{2}, \cdots, \chi_{k}$ can be found which satisfy the set of Eqs. (34) then $\epsilon$ is well defined at least to $k$ th order in the density, and is given to this order by

$$
(\epsilon-1) / 4 \pi=\sum_{j=1}^{k} \rho^{j} \chi_{i}
$$

It is clear from Eq. (34) that if $\epsilon$ is known to be well defined to $(k-1)$ th order in the density (which implies that the constants $\chi_{1}, \chi_{2}, \cdots, \chi_{k-1}$ exist and may be assumed known) then the $k$ th order investigation consists simply in determining whether there exists an additional constant $\chi_{k}$ such that

$$
\mathbf{P}_{k}(\mathbf{r})=\sum_{m=1}^{k} \chi_{m} \mathbf{E}_{k-m}(\mathbf{r})
$$

Let us begin by investigating the case $k=1$. To do this, Eq. (34) tells us that we must determine whether there exists a constant $\chi_{1}$ such that

$$
P_{1}(r)=\chi_{1} E_{0}(r) \text {. }
$$

We see immediately from Eq. (33a) that such a constant exists and that its value is

$$
\chi_{\mathbf{1}}=(1 / 3) \mu_{0}^{2} \beta \text {. }
$$

Therefore the dielectric constant is well defined at least to first order in the density. This is an ideal-gas result and is of course of a trivial nature. It is essentially an obvious consequence of the fact that in an ideal gas there is no distinction between $\mathbf{E}(\mathbf{r})$ and $\mathbf{E}_{0}(\mathbf{r})$, and is therefore of no great interest except as the first step in a more complete investigation. Note, however, that Eq. (38) is equivalent to the well-known equation

$$
\epsilon-1=(4 \pi / 3) \mu_{0}^{2} \beta \rho
$$

for the dielectric constant of an ideal gas of rigid polar molecules.

Let us therefore go on to investigate whether the dielectric constant remains well defined to second order in the density. Since we have already investigated the first-order case, Eq. (36) tells us that we need only determine whether there exists a constant $\chi_{2}$ such that

$$
\mathbf{P}_{2}(\mathbf{r})=\chi_{1} \mathrm{E}_{1}(\mathbf{r})+\chi_{2} \mathrm{E}_{0}(\mathbf{r})
$$

with $\chi_{1}$ of course given by Eq. (38). If we eliminate $\mathbf{E}_{1}(\mathbf{r})$ by means of Eq. (30) and make use of Eqs. 
(33a) and (38), Eq. (39) becomes

$$
\begin{aligned}
\mathbf{P}_{2}(\mathbf{r}) & =\left[\chi_{2}-(4 \pi / 3)\left(\mu_{0}^{2} \beta / 3\right)^{2}\right] \mathbf{E}_{0}(\mathbf{r}) \\
& +\left(\mu_{0}^{2} \beta / 3\right)^{2} \lim _{\delta \rightarrow 0} \int_{|\mathbf{r}-\mathbf{r} /|>\delta} d^{3} \mathbf{r}^{\prime} \mathbf{T}\left(\mathbf{r}-\mathbf{r}^{\prime}\right) \cdot \mathbf{E}_{0}\left(\mathbf{r}^{\prime}\right) .
\end{aligned}
$$

In order to determine whether there exists a constant $\chi_{2}$ satisfying Eq. (40), we must consider the quantity $\mathrm{P}_{2}(\mathbf{r})$, given by Eq. (33c), with some care. We first divide the sample volume $V$ into two regions (for a given value of $\mathbf{r}$ ): that which lies within a sphere of radius $\sigma^{\prime}$ centered at $\mathbf{r}$, and that which does not. Here $\sigma^{\prime}$ is a distance which is small macroscopically (i.e., much less than a typical characteristic distance over which $\mathbf{P}(\mathbf{r})$ or $\mathbf{E}_{0}(\mathbf{r})$ varies appreciably) but which may for the moment be left otherwise unspecified. The integration over $\mathbf{r}^{\prime}$ in $\mathrm{Eq}$. (33c) can then be written as the sum of integrations over these two regions, so that

$$
\mathbf{P}_{2}(\mathbf{r})=(4 \pi)^{-2} \mu_{0}^{2} \beta\left[\mathbf{I}_{S}(\mathbf{r})+\mathbf{I}_{L}(\mathbf{r})\right],
$$

where

$$
\begin{aligned}
& \mathbf{I}_{S}(\mathbf{r})=\int_{|\mathbf{r}-\mathbf{r} /|<\boldsymbol{\sigma} \prime} d^{3} \mathbf{r}^{\prime} \int d \boldsymbol{\omega}_{1} d \boldsymbol{\omega}_{2} \\
& \quad \times \exp \left\{-\beta u\left(\mathbf{r}, \boldsymbol{\omega}_{1} ; \mathbf{r}^{\prime}, \boldsymbol{\omega}_{2}\right)\right\} \mathbf{e}\left(\boldsymbol{\omega}_{1}\right) \mathbf{e}\left(\boldsymbol{\omega}_{2}\right) \cdot \mathbf{E}_{0}\left(\mathbf{r}^{\prime}\right), \\
& \mathbf{I}_{L}(\mathbf{r})=\int_{|\mathbf{r}-\mathbf{r} /|>\sigma} d^{3} \mathbf{r}^{\prime} \int d \boldsymbol{\omega}_{1} d \boldsymbol{\omega}_{2} \\
& \quad \times \exp \left\{-\boldsymbol{\beta} u\left(\mathbf{r}, \boldsymbol{\omega}_{1} ; \mathbf{r}^{\prime}, \boldsymbol{\omega}_{2}\right)\right\} \mathbf{e}\left(\boldsymbol{\omega}_{1}\right) \mathbf{e}\left(\boldsymbol{\omega}_{2}\right) \cdot \mathbf{E}_{0}\left(\mathbf{r}^{\prime}\right) .
\end{aligned}
$$

The subscripts $S$ and $L$ are meant to suggest short- and long-range, respectively. The integration variable $\mathbf{r}^{\prime}$ ranges over $V$, subject to the restrictions indicated beneath the integral signs.

We first consider $I_{S}(\mathbf{r})$. Since $\sigma^{\prime}$ is small macroscopically and $\mathbf{E}_{0}(\mathbf{r})$ is slowly varying, we can simply evaluate $\mathbf{E}_{0}\left(\mathbf{r}^{\prime}\right)$ at the point $\mathbf{r}^{\prime}=\mathbf{r}$ and bring it outside the integral

$$
\begin{aligned}
& \mathbf{I}_{S}(\mathbf{r})=\left(\int_{|\mathbf{r}-\mathbf{r} \prime|<\sigma^{\prime}} d^{3} \mathbf{r}^{\prime} \int d \boldsymbol{\omega}_{1} d \boldsymbol{\omega}_{2}\right. \\
& \left.\quad \times \exp \left\{-\beta u\left(\mathbf{r}, \boldsymbol{\omega}_{1} ; \mathbf{r}^{\prime}, \boldsymbol{\omega}_{2}\right)\right\} \mathbf{e}\left(\boldsymbol{\omega}_{1}\right) \mathbf{e}\left(\boldsymbol{\omega}_{2}\right)\right) \cdot \mathbf{E}_{0}(\mathbf{r}) .
\end{aligned}
$$

We will restrict our attention to points $\mathbf{r}$ whose distance from the surface of the sample exceeds $\sigma^{\prime}$. Since $\sigma^{\prime}$ is of molecular magnitude, this merely means that we are not concerned with the behavior of a region of negligible volume near the walls of the sample. The integration over $\mathbf{r}^{\prime}$ in Eq. (43) is therefore extended over the entire interior of the sphere $\left|\mathbf{r}-\mathbf{r}^{\prime}\right|=\sigma^{\prime}$. This spherical symmetry implies that the quantity in square brackets must be proportional to the unit tensor

$$
\begin{aligned}
& \int_{|\mathbf{r}-\mathbf{r}|<\sigma \sigma} d^{3} \mathbf{r}^{\prime} \int d \boldsymbol{\omega}_{1} d \boldsymbol{\omega}_{2} \\
& \quad \times \exp \left\{-\beta u\left(\mathbf{r}, \boldsymbol{\omega}_{1} ; \mathbf{r}^{\prime}, \boldsymbol{\omega}_{2}\right)\right\} \mathbf{e}\left(\boldsymbol{\omega}_{1}\right) \mathbf{e}\left(\boldsymbol{\omega}_{2}\right)=d(\mathbf{r}) \mathrm{U} .
\end{aligned}
$$

The coefficient $d(\mathbf{r})$ can be determined in the same way that $c(\mathbf{r})$ was determined in Sec. III; the result is

$$
\begin{aligned}
d(\mathbf{r})=(1 / 3) & \int_{\left|\mathbf{r}-\mathbf{r}^{\prime}\right|<\sigma \prime} d^{3} \mathbf{r}^{\prime} \int d \boldsymbol{\omega}_{1} d \boldsymbol{\omega}_{2} \\
& \times \exp \left\{-\boldsymbol{\beta} u\left(\mathbf{r}, \boldsymbol{\omega}_{1} ; \mathbf{r}^{\prime}, \boldsymbol{\omega}_{2}\right)\right\} \cos \gamma_{12},
\end{aligned}
$$

where $\gamma_{\mathbf{1 2}} \equiv \cos ^{-1}\left[\mathbf{e}\left(\boldsymbol{\omega}_{1}\right) \cdot \mathbf{e}\left(\boldsymbol{\omega}_{2}\right)\right]$ is the angle between the permanent moments of molecules 1 and 2. But the dependence of the pair potential on its arguments can clearly be expressed as $u\left(s, \boldsymbol{\omega}_{1}{ }^{\prime}, \boldsymbol{\omega}_{2}{ }^{\prime}\right)$, where $s \equiv\left|\mathbf{r}-\mathbf{r}^{\prime}\right|$, and $\boldsymbol{\omega}_{1}{ }^{\prime} \equiv\left(\theta_{1}{ }^{\prime}, \phi_{1}{ }^{\prime}\right)$ and $\boldsymbol{\omega}_{2}{ }^{\prime} \equiv\left(\theta_{2}{ }^{\prime}, \phi_{2}{ }^{\prime}\right)$ specify the orientation of molecules 1 and 2 relative to a coordinate system whose $z$ axis coincides with their intermolecular axis. The Jacobian of the transformation $\left(\boldsymbol{\omega}_{1}, \boldsymbol{\omega}_{2}\right) \rightarrow$ $\left(\boldsymbol{\omega}_{1}{ }^{\prime}, \boldsymbol{\omega}_{2}{ }^{\prime}\right)$ is clearly unity, so that Eq. (45) can be rewritten as

$$
\begin{aligned}
d(\mathbf{r})=(4 \pi / 3) & \int_{0}^{\sigma^{\prime}} s^{2} d s \int d \boldsymbol{\omega}_{1}^{\prime} d \boldsymbol{\omega}_{2}^{\prime} \\
& \times \exp \left\{-\beta u\left(s, \boldsymbol{\omega}_{1}^{\prime}, \boldsymbol{\omega}_{2}^{\prime}\right)\right\} \cos \gamma_{12} \equiv d,
\end{aligned}
$$

a factor of $4 \pi$ having arisen from the angular part of the integration over $\mathbf{r}^{\prime}$. We see that $d(\mathbf{r})$ is really a constant $d$, independent of $\mathbf{r}$. Given the short-range part of the pair potential (which was left arbitrary within rather wide limits in the definition of the rigiddipole model), the integral in Eq. (46) can be evaluated; it may therefore be regarded as a known constant. We now have that

$$
\mathbf{I}_{S}(\mathbf{r})=d \mathbf{E}_{0}(\mathbf{r}),
$$

with $d$ given by Eq. (46).

The integral in Eq. (46) for $d$ appears to depend strongly on $\sigma^{\prime}$, and in fact it does if $\sigma^{\prime}$ is very small. However, this integral becomes essentially independent of $\sigma^{\prime}$ if $\sigma^{\prime}$ is taken to be both $\gtrsim \sigma$ [so that Eq. (7) comes into play] and $\gtrsim 100 \AA$. Since $\sigma$ is itself small macroscopically, we can choose $\sigma^{\prime}$ in this way without violating our previous specification that $\sigma^{\prime}$ also be small macroscopically. With this choice for $\sigma^{\prime}$, it is easy to show that one incurs negligible error by writing, instead of Eq. (46),

$$
\begin{aligned}
& d=(4 \pi / 3) \int_{0}^{\infty} s^{2} d s \int d \omega_{1}{ }^{\prime} d \omega_{2}{ }^{\prime} \\
& \quad \times \exp \left\{-\beta u\left(s, \omega_{1}{ }^{\prime}, \omega_{2}{ }^{\prime}\right)\right\} \cos \gamma_{12} .
\end{aligned}
$$

The proof of this statement ${ }^{2}$ is based upon an expansion of the exponential in Eq. (48) and term by term integration in the $s$ interval $\left(\sigma^{\prime}, \infty\right)$. Since it is straightforward, we will present it in verbal outline form, omitting the details. The zero-order term in the expansion, unity, is of course prevented from causing trouble by the fact that $\int d \omega_{1}^{\prime} d \omega_{2}^{\prime} \cos \gamma_{12}=0$. One might, however, expect the linear term to give rise to a logarithmic divergence because of the long-range $s^{-3}$ radial dependence of the dipole-dipole part of the pair potential [see Eq. 
(7) ]. This divergence is prevented from being realized by the easily verified angular orthogonality relation

$$
\int d \boldsymbol{\omega}_{1}^{\prime} d \boldsymbol{\omega}_{2}^{\prime} f\left(\boldsymbol{\omega}_{1}{ }^{\prime}, \boldsymbol{\omega}_{2}{ }^{\prime}\right) \cos \gamma_{12}=0,
$$

where $s^{-3} f\left(\boldsymbol{\omega}_{1}{ }^{\prime}, \boldsymbol{\omega}_{2}{ }^{\prime}\right)$ is just the quantity $\mathbf{T}\left(\mathbf{r}-\mathbf{r}^{\prime}\right)$ : $\mathbf{e}\left(\boldsymbol{\omega}_{1}\right) \mathbf{e}\left(\boldsymbol{\omega}_{2}\right)$ expressed in terms of the variables $s, \boldsymbol{\omega}_{1}{ }^{\prime}$, and $\boldsymbol{\omega}_{2}{ }^{\prime}$. [The angular integrals in Eq. (48) must of course be performed before the $s$ (radial) integral in order for this orthogonality to save the day, but this procedure is implicit anyway in the definition of the infinite integral as the limit of a finite integral.] All higher-order terms fall off more rapidly than $s^{-3}$; they are hence purely short-ranged in character and give rise to no divergences. The orthogonality relation (49) therefore ensures that the integral in Eq. (48) converges. That it converges rapidly enough to allow the use of Eq. (48) instead of Eq. (46) then follows immediately from the fact that our choice of $\sigma^{\prime}$ makes the relevant dimensionless parameter $\mu_{0}^{2} \beta /\left(\sigma^{\prime}\right)^{3}$ typically much less than unity (in fact, only about $10^{-4}$ ) at ordinary temperatures.

Let us now go on to consider $\mathbf{I}_{L}(\mathbf{r})$, given by $\mathbf{E q}$. (42b). Since we have chosen $\sigma^{\prime} \gtrsim \sigma$, we can by virtue of Eq. (7) write

$$
\begin{aligned}
& \mathbf{I}_{L}(\mathbf{r})=\int_{\mid \mathbf{r}-\mathbf{r} />\sigma} d^{3} \mathbf{r}^{\prime} \int d \boldsymbol{\omega}_{1} d \boldsymbol{\omega}_{2} \\
& \times \exp \left\{\mu_{0}^{2} \beta \mathbf{T}\left(\mathbf{r}-\mathbf{r}^{\prime}\right): \mathbf{e}\left(\boldsymbol{\omega}_{1}\right) \mathbf{e}\left(\boldsymbol{\omega}_{2}\right)\right\} \mathbf{e}\left(\boldsymbol{\omega}_{1}\right) \mathbf{e}\left(\boldsymbol{\omega}_{2}\right) \cdot \mathbf{E}_{0}\left(\mathbf{r}^{\prime}\right) .
\end{aligned}
$$

But because $\mu_{0}^{2} \beta /\left(\sigma^{\prime}\right)^{3} \ll 1$, we can expand the exponential in Eq. (50) and retain only the lowest-order nonzero term. Since $\int d \boldsymbol{e}(\boldsymbol{\omega})=0$, this is the linear term

$$
\begin{aligned}
\mathbf{I}_{L}(\mathbf{r}) & =\mu_{0}^{2} \beta \int_{|\mathbf{r}-\mathbf{r} /|>\sigma^{\prime}} d^{3} \mathbf{r}^{\prime} \int d \omega_{1} d \boldsymbol{\omega}_{2} \\
\times & {\left[\mathbf{T}\left(\mathbf{r}-\mathbf{r}^{\prime}\right): \mathbf{e}\left(\boldsymbol{\omega}_{1}\right) \mathbf{e}\left(\boldsymbol{\omega}_{2}\right)\right] \mathbf{e}\left(\boldsymbol{\omega}_{1}\right) \mathbf{e}\left(\boldsymbol{\omega}_{2}\right) \cdot \mathbf{E}_{0}\left(\mathbf{r}^{\prime}\right) } \\
& =(4 \pi / 3)^{2} \mu_{0}^{2} \beta \int_{|\mathbf{r}-\mathbf{r} /|>\sigma^{\prime}} d^{3} \mathbf{r}^{\prime} \mathbf{T}\left(\mathbf{r}-\mathbf{r}^{\prime}\right) \cdot \mathbf{E}_{0}\left(\mathbf{r}^{\prime}\right),
\end{aligned}
$$

where we have made use of the elementary fact that $\int d \boldsymbol{\omega} \mathbf{e}(\boldsymbol{\omega}) \mathbf{e}(\boldsymbol{\omega})=(4 \pi / 3) \cup$. Combining Eqs. (41), (47), and (51), we obtain

$$
\begin{aligned}
\mathbf{P}_{2}(\mathbf{r})= & (4 \pi)^{-2} \mu_{0}^{2} \beta d \mathbf{E}_{0}(\mathbf{r}) \\
& +\left(\mu_{0}^{2} \beta / 3\right)^{2} \int_{|\mathbf{r}-\mathbf{r} \prime|>\sigma} d^{3} \mathbf{r}^{\prime} \mathbf{T}\left(\mathbf{r}-\mathbf{r}^{\prime}\right) \cdot \mathbf{E}_{0}\left(\mathbf{r}^{\prime}\right) .
\end{aligned}
$$

This is our final result for $\mathbf{P}_{2}(\mathbf{r})$, which we must compare with Eq. (40). We recall that the limit $\delta \rightarrow 0$ in Eq. (40) means that $\delta$ becomes macroscopically small but remains microscopically large. Since this is precisely the range in which $\sigma^{\prime}$ has been chosen to lie, the limit can be achieved simply by setting $\delta=\sigma^{\prime}$ in Eq. (40). When this is done, Eqs. (40) and (52) become identical if we make the identification

$$
(4 \pi)^{-2} \mu_{0}^{2} \beta d=\chi_{2}-(4 \pi / 3)\left(\mu_{0}^{2} \beta / 3\right)^{2} .
$$

We see, therefore, that there does in fact exist a $\chi_{2}$ such that Eq. (40) is true, and that its value is

$$
\chi_{2}=(4 \pi)^{-2} \mu_{0}^{2} \beta d+(4 \pi / 3)\left(\mu_{0}^{2} \beta / 3\right)^{2},
$$

with $d$ given by Eq. (48). Therefore $\epsilon$ exists at least to second order in the density, and to this order is given by

$$
(\epsilon-1) / 4 \pi=\chi_{1} \rho+\chi_{2} \rho^{2},
$$

with $\chi_{1}$ and $\chi_{2}$ given by Eqs. (38) and (53), respectively.

It is of intrest to re-express $\mathrm{Eq}$. (54) in terms of the "Clausius-Mossotti function" $(\epsilon-1) /[(\epsilon+2) \rho]$, since this is the quantity most frequently dealt with in earlier dielectric theories. It is easy to show that Eq. (54) is equivalent, within terms of order $\rho^{3}$, to

where

$$
\rho^{-1}(\epsilon-1) /(\epsilon+2)=A_{\mathrm{CM}}+B_{\mathrm{CM}} \rho
$$

$$
\begin{aligned}
A_{\mathrm{CM}}= & (4 \pi / 3) \chi_{1}=(4 \pi / 9) \mu_{0}{ }^{2} \beta \\
B_{\mathrm{CII}}= & (4 \pi / 3)\left[\chi_{2}-(4 \pi / 3) \chi_{1}{ }^{2}\right]=(4 \pi)^{-1}\left(\mu_{0}{ }^{2} \beta / 3\right) d \\
= & \frac{1}{9}\left(\mu_{0}^{2} \beta\right) \int_{0}^{\infty} s^{2} d s \int d \omega_{1}{ }^{\prime} d \omega_{2}{ }^{\prime} \\
& \quad \times \exp \left\{-\beta u\left(s, \omega_{1}{ }^{\prime}, \omega_{2}{ }^{\prime}\right)\right\} \cos \gamma_{12},
\end{aligned}
$$

and use has been made of Eqs. (38), (53), and (48). The Clausius-Mossotti function, of course, like the dielectric constant itself, has only been shown to be well defined to second order in $\rho$. The coefficients $A_{\mathrm{CM}}$ and $B_{\mathrm{CM}}$ are sometimes called the first and second dielectric virial coefficients. Our Eqs. (56) for these quantities are identical to results obtained long ago by Buckingham and Pople. ${ }^{16}$ However, we have obtained them here as a by-product of our demonstration that the quantity $\epsilon$ is well defined to second order in $\rho$, while Buckingham and Pople obtained them under the usual assumption that $\epsilon$ is well defined. Since we have verified this assumption to second order in $\rho$, our results must agree with theirs if no error has been made, and it is comforting to see that they do.

We remark that in conventional theories of the dielectric constant (and in particular in Buckingham and Pople's work) the occurrence of the ClausiusMossotti function (rather than some other rational function of $\epsilon$ ) seems to be a direct consequence of the usual choice of a spherical sample and a uniform external field $\mathbf{E}_{0}$. Here, however, we have obtained Eqs. (55) and (56) by consideration of a sample of arbitrary shape in an arbitrary slowly-varying positiondependent external field $\mathbf{E}_{0}(\mathbf{r})$. We are therefore assured that at least to second order in $\rho$ the effects of sample shape and nonuniform external field are taken precisely into account by Eq. (3). 


\section{CONCLUDING REMARKS}

The demonstration of the preceding section is interesting apart from its result, for it provides some valuable insight into the connection between long-range dipolar effects on the macroscopic and molecular levels. It is illuminating to trace the path of the dipole tensor $\mathbf{T}\left(\mathbf{R}_{1}-\mathbf{R}_{2}\right)$, which originates in the dipole-dipole part of the intermolecular potential and which ultimately emerges in correspondence to the dipole tensor $\mathbf{T}\left(\mathbf{r}-\mathbf{r}^{\prime}\right)$ in the purely macroscopic Eq. (1). This correspondence is precisely that which is necessary to ensure that the nonlocal relation (18) between $\mathbf{P}(\mathbf{r})$ and $\mathbf{E}_{0}(\mathbf{r})$ is equivalent to the local relation (3) between $\mathbf{P}(\mathbf{r})$ and $\mathbf{E}(\mathbf{r})$. It is clear from our derivation that the molecular origin of macroscopic long-range effects in polar substances is the linear term in the expansion of the Boltzmann factor $\exp \left\{\mu_{0}^{2} \beta \mathbf{T}\left(\mathbf{R}_{i}-\mathbf{R}_{j}\right): \mathbf{e}\left(\boldsymbol{\omega}_{i}\right) \mathbf{e}\left(\boldsymbol{\omega}_{j}\right)\right\}$. This is the only term in the expansion which has the long-range $\left|\mathbf{R}_{\boldsymbol{i}}-\mathbf{R}_{j}\right|^{-3}$ radial dependence, and it is hence the term which is responsible for any long-range effects or divergences in the problem. Although this term is sometimes prevented from contributing (or causing a divergence) by such angular orthogonality relations as Eq. (49), we have seen that in other cases it is of primary importance.

The result of our demonstration, namely that $\epsilon$ is well defined at least to second order in $\rho$, is also of interest; it constitutes, to our knowledge, the first theoretical indication that Eq. (3) is other than qualitatively valid for polar fluids under any circumstances. However, although nontrivial this result must still be considered as something of a special case. According to Eq. (24) it is the behavior of $\rho^{(2)}$ which is of central importance to dielectric theory, and to second order in $\rho$ the calculation of this quantity involves only two interacting molecules, with no intervening medium between them to modify their net interaction. This intervening medium certainly plays an important role in the dielectric behavior of dense systems, and it would therefore be of interest to extend the investigation of Sec. $V$ at least to third order in $\rho$. It is clear in principle how to do this, but in practice one rapidly bogs down in the algebra. We hope to be able to streamline the procedure in order to carry out the third-order investigation at some future time. In any case, however, it is clear that an investigation using density expansions is not relevant for liquids, since the expansions cannot then reasonably be expected to converge. We are therefore still a long way from knowing whether the dielectric constant is well defined for polar liquids. To investigate this question, some approximate means of taking the long-range orientational correlations into account must be developed.

Previous efforts to take these long-range correlations and their effects (in particular, shape-dependence of molecular averages) into account in dielectric the- ory $y^{4-7,9,12}$ have apparently all been carried out within the framework of the assumption that $\epsilon$ is well defined. Since the validity of this assumption depends critically on the precise nature of these long-range correlations, such a procedure is of questionable legitimacy. We feel strongly that the nature of these long-range correlations should be studied apart from the assumption that $\epsilon$ is well defined. Once a satisfactory method of approximating them is found, the results of Sec. III can be used to investigate the validity of this assumption.

A final point: One might at first have thought that to investigate the validity of Eq. (3), it would be necessary to express the macroscopic Maxwell electric field $\mathbf{E}(\mathbf{r})$ [in addition to $\mathbf{P}(\mathbf{r})$ ] as a statistical mechanical average of some microscopic dynamical variable. The question of how to do this is a somewhat delicate one $^{1,2}$ [it is related to the dependence of the integral in Eq. (1) on the shape of the cavity], and it is therefore with relief that we see it can be completely bypassed. It is clear from our second-order investigation that the validity of $\mathbf{E q}$. (3) can be investigated in a straightforward manner without considering $\mathbf{E}(\mathbf{r})$ as anything other than the purely macroscopic quantity given by Eq. (1).

\section{APPENDIX}

We want here to express the pair distribution function $\rho^{(2)}$ as a power series in the density. This expansion is of course well known for molecules interacting via short-ranged forces, ${ }^{21}$ but the long-range nature of the permanent dipole-dipole interaction renders the validity of these usual results uncertain for polar substances. However, the author has recently developed a densityexpansion method ${ }^{22}$ which makes no explicit shortrange-forces assumption, and which is therefore applicable to polar substances, even when shape-dependent effects are present. This method expresses the thermodynamic limit $F(\rho, T)$ of a canonical statisticalmechanical average $F(N, V, T)$ as

$$
F(\rho, T)=\sum_{k=0}^{\infty} A_{k}(T) \rho^{k},
$$

where the coefficients $A_{k}(T)$ are given by

$$
\begin{array}{r}
A_{k}(T)=(-1)^{k} \lim _{V \rightarrow \infty}\left\{V^{k} \sum_{j=0}^{k}(-1)^{j}[j !(k-j) !]^{-1}\right. \\
\times F(j, V, T)\} .
\end{array}
$$

Although these results are strictly applicable only in the limit of an infinite system, they may be applied with negligible error to finite but macroscopic systems except in rare cases.

We shall identify $F(N, V, T)$ with the quantity $\rho^{(2)}$ 
given by Eq. (23),

$$
\begin{aligned}
F(N, V, T) & =\rho^{(2)}\left(\mathbf{Q}_{1}, \mathbf{Q}_{2}\right) \\
& =N(N-1) \frac{\int d \mathbf{Q}^{N-2} \exp \left\{-\beta U_{0}\left(\mathbf{Q}^{N}\right)\right\}}{\int d \mathbf{Q}^{N} \exp \left\{-\beta U_{0}\left(\mathbf{Q}^{N}\right)\right\}} .
\end{aligned}
$$

To go out to second order in $\rho, \mathrm{Eq}$. (A2) tells us that we need the quantities $F(0, V, T), F(1, V, T)$, and $F(2, V, T)$; these are obtained by setting $N=0,1$, and 2 in Eq. (A3). We find that $F(0, V, T)=F(1, V, T)=0$, and that

$$
\begin{aligned}
& F(2, V, T) \\
& =2 \exp \left\{-\beta u\left(\mathbf{Q}_{1}, \mathbf{Q}_{2}\right)\right\} / \int d \mathbf{Q}_{1} d \mathbf{Q}_{2} \exp \left\{-\beta u\left(\mathbf{Q}_{1}, \mathbf{Q}_{2}\right)\right\}
\end{aligned}
$$

Equation (A2) then yields for the first three $A_{k}(T)$,

$$
\begin{gathered}
A_{0}(T)=A_{\mathbf{1}}(T)=0, \\
A_{2}(T)=\lim _{V \rightarrow \infty}\left[V^{2} \frac{\exp \left\{-\beta u\left(\mathbf{Q}_{1}, \mathbf{Q}_{2}\right)\right\}}{\int d \mathbf{Q}_{1} d \mathbf{Q}_{2} \exp \left\{-\beta u\left(\mathbf{Q}_{1}, \mathbf{Q}_{2}\right)\right\}}\right] .
\end{gathered}
$$

But

$$
\begin{aligned}
\int d \mathbf{Q}_{1} d \mathbf{Q}_{2} \exp \left\{-\beta u\left(\mathbf{Q}_{\mathbf{1}}, \mathbf{Q}_{2}\right)\right\}=(4 \pi)^{2} V^{2} \\
\quad+\int d \mathbf{Q}_{\mathbf{1}} d \mathbf{Q}_{\mathbf{2}}\left[\exp \left\{-\beta u\left(\mathbf{Q}_{\mathbf{1}}, \mathbf{Q}_{\mathbf{2}}\right)\right\}-1\right],
\end{aligned}
$$

and the integral on the right-hand side of $\mathrm{Eq}$. (A7) is of a short-ranged nature, in spite of the long-range dipole-dipole potential, because of the fact that $\int d \boldsymbol{\omega}_{1} d \boldsymbol{\omega}_{2} \mathbf{T}\left(\mathbf{R}_{1}-\mathbf{R}_{2}\right): \mathbf{e}\left(\boldsymbol{\omega}_{1}\right) \mathbf{e}\left(\boldsymbol{\omega}_{2}\right)=0$. This relation plays a role analogous to that of Eq. (49) in the development of Sec. V; it prevents the linear term in the expansion of the exponential (which is, as discussed in Secs. V and VI, the only term which can give rise to a divergence or to long-range behavior) from contributing to the integral when $\left|\mathbf{R}_{\mathbf{1}}-\mathbf{R}_{\mathbf{2}}\right|>\sigma$ [see Eq. (7)]. If $V^{1 / 3} \gg \sigma$, therefore, we can write with negligible error

$$
\begin{aligned}
& \int d \mathbf{Q}_{1} d \mathbf{Q}_{2}\left[\exp \left\{-\beta u\left(\mathbf{Q}_{1}, \mathbf{Q}_{2}\right)\right\}-1\right] \\
& =4 \pi V \int_{0}^{\infty} s^{2} d s \int d \omega_{1}{ }^{\prime} d \boldsymbol{\omega}_{2}{ }^{\prime}\left[\exp \left\{-\beta u\left(s, \boldsymbol{\omega}_{1}{ }^{\prime}, \boldsymbol{\omega}_{2}{ }^{\prime}\right)\right\}-1\right] .
\end{aligned}
$$

Combining Eqs. (A7) and (A8) with Eq. (A6) and taking the limit, we obtain

$$
A_{2}(T)=(4 \pi)^{-2} \exp \left\{-\beta u\left(\mathrm{Q}_{1}, \mathrm{Q}_{2}\right)\right\} .
$$

Because of Eq. (A5), the expansion of $\rho^{(2)}\left(\mathbf{Q}_{1}, \mathbf{Q}_{2}\right)$ begins with the second-order term. The result (A9) for the coefficient $A_{2}(T)$ is of course just the usual result, ${ }^{21}$ ordinarily obtained under the assumption that the intermolecular pair potential $u\left(\mathbf{Q}_{\mathbf{1}}, \mathbf{Q}_{2}\right)$ is purely short-ranged in nature. We therefore see that this usual result is not modified by the long-range nature of the dipole-dipole potential, but this fact could not have been anticipated beforehand with any certainty.

* This work was presented in the author's Ph.D. thesis (Massachusetts Institute of Technology, 1970, unpublished) and was supported by the National Science Foundation. Its preparation for publication has been supported by the Air Force Office of Scientific Research through the National Research Council.

† National Science Foundation Graduate Fellow 1966-1970.

Air Force Office of Scientific Research-National Research Council

Postdoctoral Research Associate 1970-1971. Address after 15

September 1971: Department of Physics, The University of

Utah, Salt Lake City, Utah 84112.

${ }^{1}$ W. F. Brown, Jr., in Handbuch der Physik, edited by S. Flugge (Springer, Berlin, 1956), Vol. 17, p. 1.

${ }^{2}$ J. D. Ramshaw, Ph.D. thesis, Massachusetts Institute of Technology, Cambridge, Mass., 1970.

${ }^{3}$ Unfortunately, there does not appear to be any direct way to experimentally measure $\mathbf{P}(\mathbf{r})$ as a function of $\mathbf{r}$.

${ }^{4}$ J. G. Kirkwood, J. Chem. Phys. 7, 911 (1939).

${ }^{5}$ H. Frohlich, Theory of Dielectrics (Oxford U. P., Oxford, 1957), 2nd ed.

${ }^{6}$ F. E. Harris and B. J. Alder, J. Chem. Phys. 21, 1031 (1953).

7 F. E. Harris, J. Chem. Phys. 23, 1663 (1955).

${ }^{8}$ A. D. Buckingham and J. A. Pople, Trans. Faraday Soc. 51,1029 (1955)

9 A. D. Buckingham, Proc. Roy. Soc. (London) A238, 235 (1956).

${ }^{10}$ R. H. Cole, J. Chem. Phys. 27, 33 (1957).

11 M. Mandel and P. Mazur, Physica 24, 116 (1958).

12 J. D. Ramshaw, D. W. Schaefer, J. S. Waugh, and J. M. Deutch, J. Chem. Phys. 54, 1239 (1971).

13 See for example P. Mazur, Advan. Chem. Phys. 1, 309 (1958); R. K. Bullough, J. Phys. A 1, 409 (1968) ; 2, 477 (1969); 3, 708, 726, 751 (1970).

${ }^{14}$ H. A. Lorentz, The Theory of Electrons (Dover, New York, 1952), 2nd ed.

${ }^{15}$ See for example R. Rosenberg and M. Lax, J. Chem. Phys. 21, 424 (1953) ; V. Ambegaokar and W. Kohn, Phys. Rev. 117, 423 (1960); V. Ambegaokar, ibid. 121, 91 (1961); M. A. Ball and A. D. McLachlan, Proc. Roy. Soc. (London) A282, 433 (1964).

${ }^{16}$ A. D. Buckingham and J. A. Pople, Trans. Faraday Soc. 51, 1179 (1955).

${ }_{17}$ We are omitting ferroelectric materials (which are invariably solids anyway) from our consideration.

${ }_{18}$ R. Kubo, J. Phys. Soc. Japan 12, 570 (1957).

${ }_{19}$ The term "long-range" here refers to the fact that the $r^{-3}$ radial dependence of the dipole-dipole potential gives rise to a logarithmic divergence in a three-dimensional integral over all space. This means, among other things, that in a uniform distribution of dipoles the effect at a given point of far-away dipoles cannot be neglected in comparison to that of nearby dipoles.

${ }^{20}$ See Ref. 2, Appendix B, and references cited therein.

${ }^{21}$ I. Oppenheim and P. Mazur, Physica 23, 197 (1957).

${ }^{22}$ J. D. Ramshaw, "A New Method for Generating Density"

Expansions," J. Chem. Phys. (to be published). 